\title{
Water-water and ion-water hydrogen bonding in sulfuric acid solutions
}

\author{
Manel Canales ${ }^{\mathrm{a})}$ and Elvira Guàrdia
}

Departament de Física, Universitat Politècnica de Catalunya, Campus Nord-Edifici B4-B5, Jordi Girona 1-3, Barcelona E-08034, Spain.

\footnotetext{
a) Author to whom correspondence should be addressed. Electronic mail: manel.canales@upc.edu
} 


\begin{abstract}
Molecular dynamics simulations of sulfuric acid solutions at room temperature and at concentrations up to $40 \mathrm{wt} \%$ have been performed. The dissociation of the acid in water has been implicitly taken into account by assuming that the simulated systems are made up of the following molecular constituent species: water molecules, hydronium cations, bisulfate anions and sulfate dianions. Calculations have been carried out using a reliable force field which provides a good agreement between the experimental and the simulation densities and viscosity coefficients. The density and several structural and dynamical quantities, such as the radial distribution functions, the shear viscosity and the diffusion coefficients, have been calculated. The results show a noticeable good agreement with the available experimental data. A study of the hydrogen bonding which involve water molecules has also been performed. Then, the mean number of hydrogen bonds between water molecules and between ions and water molecules, and the percentages of these hydrogen bonds per water molecule or ion have been computed. Moreover, an analysis of the formation and rupture of the hydrogen bonds has also been made. To this end, the continuous and the interrupted hydrogen bond lifetimes have been calculated. Our findings suggest that the water molecules hydrogen bonded to the anions are more labile than those bonded to other water molecules or to hydronium ions. Finally, the role played by the acid concentration on these quantities has also been analyzed. Then, for instance, an increase of the lability of the water molecules, which are hydrogen bonded to other water molecules, has been observed when the concentration rises.
\end{abstract}




\section{KEYWORDS}

1) Sulfuric acid solutions

2) Molecular dynamics simulation.

3) Structural properties.

4) Viscosity and diffusion.

5) Hydrogen bonds.

6) Lability. 


\section{INTRODUCTION}

Sulfuric acid $\left(\mathrm{H}_{2} \mathrm{SO}_{4}, \mathrm{SA}\right)$ is one of the most widely used commodity chemicals. In fact, some authors stated that its production and consumption are an indicator of the industrial power of a country. ${ }^{1} \mathrm{SA}$ is mainly employed to produce fertilizers, but it is also used in the manufacture of chemicals, petroleum refining, processing of metals, etc. ${ }^{2}$ The study of aqueous SA solutions is important because, for instance, dilute SA solutions are the common electrolyte in metal electrowinning and electroplating, ${ }^{3}$ concentrations up to $40 \mathrm{wt} \%$ are the usual electrolyte of the lead-acid batteries ${ }^{4}$ and because sulfate aerosols can be considered as water and SA mixtures up to $80 \mathrm{wt} \%{ }^{5}$

SA dissociates in water following two different steps:

$$
\begin{gathered}
\mathrm{H}_{2} \mathrm{SO}_{4}+\mathrm{H}_{2} \mathrm{O} \rightleftarrows \mathrm{HSO}_{4}^{-}+\mathrm{H}_{3} \mathrm{O}^{+} \\
\mathrm{HSO}_{4}^{-}+\mathrm{H}_{2} \mathrm{O} \rightleftarrows \mathrm{SO}_{4}^{2-}+\mathrm{H}_{3} \mathrm{O}^{+}
\end{gathered}
$$

The first process is complete at room temperature and concentrations up to $40 \mathrm{~mol} / \mathrm{kg}$. However, the second depends on the concentration and the temperature. ${ }^{6}$ In any case, the constituent molecular species which can be found in any SA aqueous solution are: $\mathrm{H}_{2} \mathrm{O}$, hydronium ions $\mathrm{H}_{3} \mathrm{O}^{+}$, bisulfate ions $\mathrm{HSO}_{4}^{-}$and sulfate dianions $\mathrm{SO}_{4}^{2-}$.

Computer simulation studies of aqueous SA solutions, using classical and quantum mechanical methods, have been performed in the last two decades. For instance, Monte Carlo simulations have been used to study the binary nucleation of SA-water systems. Then, Kusaka et al. ${ }^{7}$ got information about the clusters shape and the dissociation of the SA molecules inside the clusters. Kathmann et al. ${ }^{8}$ derived effective atom-atom pair potentials suitable to be used in simulations of small SA clusters. Particularly, they employed them to compute the cluster's free energy of formation. Classical molecular dynamics (MD) simulations have also been used to study SA-water clusters. Then, Ding et $a .^{9}$ analyze the distribution of waters around SA molecules at atmospheric 
conditions. Toivola et al. ${ }^{10}$ studied the structure of the clusters at several concentrations and Matsubara et al. ${ }^{11}$ the dependence of the nucleation rate in terms of the concentration. Subsequently, Ishiyama et al. ${ }^{12}$ performed MD simulations of aqueous SA solutions to compute the sum frequency generation spectrum. These studies have validated the reliability of several force fields, which in many cases, incorporates the ionization of the SA and water molecules. Several first-principles simulations of aqueous SA solutions had also been performed. Then, Choe et al. ${ }^{13}$ carried out simulations to compute structural, dynamical and electrical properties at several concentrations. Wan et al. ${ }^{14}$ analyzed the degree of dissociation of SA and the electronic structure in a dilute solution. Hammerich et al. ${ }^{15}$ studied the SA ionization and the proton hopping between the bisulfate ion $\mathrm{HSO}_{4}{ }^{-}$oxygen sites corresponding to a low concentrated solution. Finally, ab initio simulations of $\mathrm{HSO}_{4}^{-}$and $\mathrm{SO}_{4}^{2-}$ ions in aqueous solutions have also been performed to analyze structural and dynamical properties. ${ }^{16,17}$

Hydrogen bonds (HB) are short range nonbonded interactions which occur in many chemical and biological systems. ${ }^{18,19}$ Simulations of water and other hydrogen-bonded liquids have been carried out during the last five decades. ${ }^{20}$ Usually, the mean number of HB per molecule, their lifetimes and the percentages of molecules with " $\mathrm{n}$ " HB are computed. These properties allow us to understand the structure and dynamics of the simulated systems. For instance, it is thanks to this type of detailed information that we know that water shows a topologically complex three-dimensional tetrahedral network of $\mathrm{HB}^{21}$ It is well known that every SA molecule has two acceptors (the oxygen atoms of the sulfonyl group) and two donors (the oxygen atoms of the hydroxyl group). Recently, Canales and Guàrdia ${ }^{22}$ have carried out a MD study of pure SA. They concluded that it has a HB network which resembles the one of water. Moreover, they 
also found that SA and water have similar short labilities. That is to say, the HB break and reform quickly.

The first objective of this work is to look for reliable force fields for aqueous SA solutions at room temperature and for a wide range of concentrations. To this end, thermodynamic, structural and dynamical properties, such as the density, the viscosity and the radial distribution functions will be calculated and compared with the available experimental data. The second goal is to perform a HB analysis focused on the water molecules. To this end, four different types of HB will be separately studied: waterwater, bisulfate ion-water, sulfate dianion-water and hydronium ion-water. In each case, the mean number of $\mathrm{HB}$, their lifetimes and the percentages of hydrogen bonds per water molecule or ion will be computed. Special attention will be paid on their concentration dependence.

The paper is organized as follows: Simulation details are explained in the next section, the Results and Discussion section is devoted to the exposition of the structural, dynamical and HB analyses. The concluding remarks will be summarized in the last section.

\section{METHODOLOGY}

\section{A. Equilibrium geometry data}

The four constituent molecular species $\mathrm{H}_{2} \mathrm{O}, \mathrm{H}_{3} \mathrm{O}^{+}, \mathrm{HSO}_{4}^{-}$and $\mathrm{SO}_{4}^{2-}$ are shown in Figure 1. Their equilibrium bond lengths and bending bond angles, which are gathered in Tables I and II, have been compiled from the literature. The $\mathrm{HSO}_{4}^{-}$values are those obtained by Arstila et al. ${ }^{23}$ from ab initio calculations of SA with 1-3 water molecules. The $\mathrm{SO}_{4}^{2-}$ parameters are those computed by Ishiyama et $a{ }^{12}$ from ab initio calculations. The hydronium and water data are respectively those used by Kusaka et 
$a l^{7}$ in their Monte Carlo simulation study of the binary nucleation of the SA-water system and the ones of the well-known SPCE model. ${ }^{24}$

\section{B. Force fields and computer simulation details}

MD simulations have been carried out using the GROMACS 4.6 software package. ${ }^{25}$ As bond lengths have been constrained using the SHAKE algorithm, ${ }^{26}$ the intramolecular force field only has bending and dihedral terms. The bond bending interactions have been computed using the OPLS harmonic angle functional force field $^{27} V_{\text {bend }}(\theta)=\frac{1}{2} k_{\theta}\left(\theta-\theta_{e q}\right)^{2}$.The equilibrium bond lengths and the bending angles are those displayed in Tables I and II. The interactions involving sulfur atoms have been computed using the $k_{\theta}$ values recently used by Canales and Guàrdia ${ }^{22}$ in their computer simulation study of SA. These data are based on those developed by Ketko et al. ${ }^{28}$ in their simulation study of sulfur dioxide and the ones proposed by Yosa and Meuwly $^{29}$ to analyze the vibrational induced dissociation of SA. The hydronium ion bending interactions have been obtained using the $k_{\theta}$ parameter of the SPC flexible water model ${ }^{30}$. At last, we turn to the torsional contribution, which is only starred by the $\mathrm{HSO}_{4}^{-}$ion. Specifically, in this case the H-O-S-O' dihedral interaction shows three minima located at $-120^{\circ}, 0^{\circ}$ and $120^{\circ}$. It has been modeled using the functional form proposed by Ryckaert and Bellemans ${ }^{31}: V_{\text {dine }}(\varnothing)=\sum_{n=0}^{5} C_{n} \cos ^{n}(\varnothing-\pi)$. The parameters are collected in Table III.

The van der Waals interactions have been established using the Lennard-Jones 12-6 potential. The homoatomic pair parameters of the OPLS-AA force field, ${ }^{32}$ which are gathered in Table IV, have been used. The heteroatomic pair parameters have been calculated using the Lorentz Berthelot mixing rules. It is important to note that the hydronium ions have been simulated using the SPCE Lennard-Jones parameters and not 
those proposed by Kusaka et al. ${ }^{7}$, because simulations using the former set render a better agreement with the experimental data.

Four different aqueous SA solutions have been simulated: 1.13, 2.55, 4.37 and 6.79 m. That is to say $10,20,30$ and $40 \mathrm{wt} \%$. The number of molecules of the constituent species have been compiled in Table V. They have been calculated assuming that SA dissociates in water following two processes. In the former the dissociation is complete, but in the second it depends on a concentration and temperature dependent dissociation constant, which has been experimentally determined by Knopf et al. ${ }^{6}$

Simulations have been carried out at $298 \mathrm{~K}$ and $1 \mathrm{~atm}$, employing the usual periodic boundary conditions with a 1 fs integration time step. The Berendsen weak coupling pressure and temperature bath algorithm ${ }^{33}$ have been used to fit the temperature and the pressure. To this end, the time constant of the heat bath coupling and the pressure relaxation times were, respectively, 0.1 and 1 ps. The van der Waals interactions have been truncated at a cutoff distance of $1 \mathrm{~nm}$. The electrostatic interactions have been computed using the well-known Ewald summation methodology. ${ }^{34}$ Then, the real space term was truncated at the van der Waals distance cut-off. Moreover, the reciprocal space contribution has been computed using an interpolation procedure which employs an infinite grid of points (Particle Mesh Ewald), ${ }^{35}$ the grid spacing being $0.12 \mathrm{~nm}$.

The equilibrated starting structures have been obtained adopting the following methodology: a first configuration has been generated introducing at random the molecules inside a cubic box of length $4 \mathrm{~nm}$. Later, the system has been relaxed after $10^{4}$ steps of an energy minimization procedure, which uses a steepest descent algorithm. ${ }^{36}$ Finally, $10^{7}$ time steps have been carried out to equilibrate the system. The properties have been calculated from 10 ns time MD runs series. In all cases the coordinates and velocities have being saved every 50 steps. 
The electrostatic contributions have been calculated using the set of partial charges collected in Table VI. They have been chosen in such a way that a good agreement is obtained between the experimental $^{37,38}$ and the simulation results for the density and the viscosity. It should be noted that the charge values are different to those employed by Canales and Guàrdia ${ }^{22}$ in their work on pure SA and those used by Artsila et al. ${ }^{23}$ and Ding et $a l .{ }^{9}$ to study several SA-water systems. It is important to notice that we have also been carried out simulations with partial charge values similar to those used in reference 22. However, the viscosity results obtained using them were clearly much larger than the experimental data, particularly for acid concentrations larger than $40 \%$ wt.

The viscosity has been calculated using the periodic perturbation method. ${ }^{39}$ In short, a periodic external force, which depends on the z-direction coordinate, is applied to all the particles in the x-direction. As a result, a velocity field is induced in the system, which satisfies the Navier-Stokes equation. The viscosity is directly related to the velocity profile. It is important to note that this relationship strongly depends on the external acceleration amplitude $\lambda$. Therefore, in one hand, $\lambda$ should be small enough so that the perturbation does not significantly modify the equilibrium condition and, on the other hand, small forces induce to low signal-to-noise ratio. The key of the method is to use a suitable set of small $\lambda$ values and to extrapolate the results to zero following a linear fit procedure. The results are displayed in Figure 2.

As it has been previously stated, a set of charge values has been chosen in order to achieve a good agreement between the experimental ${ }^{37,38}$ and the simulation density and viscosity results. These data have been gathered in Table VII at the range of concentrations considered in this work. An excellent agreement has been observed between the experimental and simulation density findings. However, the comparison 
between the viscosities gives worse results and deserves a special comment. Then, the viscosity coefficient of pure water at $298 \mathrm{~K}$ calculated by MD using the SPCE model ${ }^{41}$ is $0.729 \mathrm{cP}$. This result differs significantly from the experimental one ${ }^{40} 0.89 \mathrm{cP}$. Therefore, for diluted solutions, the discrepancies between experimental and simulation results can be mainly attributed to the poor MD findings obtained using the SPCE model. In addition, the MD simulation results of aqueous SA solutions at concentrations larger than 50 wt \% disagree with experimental data by more than $100 \%$. For this reason, in this work we have only performed MD simulations of aqueous SA solutions at concentrations up to $40 \mathrm{wt} \%$.

\section{RESULTS AND DISCUSSION}

\section{A. Structural properties}

Our study has been focused on the analysis of the distribution of water molecules around other water molecules and around the three types of ions species. To distinguish the oxygen and hydrogen atoms of different species we have used the following notation: OW, HW denote respectively the oxygen and hydrogen atoms of water, OB denotes oxygen atoms of a bisulfate ion, OS denotes oxygen atoms of a sulfate dianion, and O3, H3 denote respectively the oxygen and hydrogen atoms of an hydronium ion.

\section{A.1 Water-water}

Some years ago, Mark et al. ${ }^{42}$ made a comparative MD study of several dynamical and structural properties of water at room temperature employing five well-known models. Particularly, they determined the $g_{O W-O W}(r)$ and $g_{O W-H W}(r)$ partial radial distribution functions using the SPCE model. According to them, $g_{\text {ow-Ow }}(r)$ exhibits three maxima located at $0.275,0.45$ and $0.685 \mathrm{~nm}$ and two minima at 0.335 and 0.568 nm. Moreover, they also found that $g_{O W-H W}(r)$ has two maxima located at 0.177 and $0.325 \mathrm{~nm}$ and one minimum at $0.241 \mathrm{~nm}$. These results are in a good agreement with 
our findings at $1.13 \mathrm{~m}$, which have been displayed in Figure 3. In addition, the second and third $g_{\text {Ow-Ow }}(r)$ peaks slightly shift to smaller distances when the acid concentration increases. Particularly, the second peak of the $6.79 \mathrm{~m}$ solution almost collapses, denoting that water becomes less structured. Gallo et al. ${ }^{43}$ have been observed a similar behavior in their MD study of potassium halides. On the contrary, the second $g_{O W-H W}(r)$ peak increases when the concentration raises. These results are consistent with those obtained by Wann et al. ${ }^{14}$ for dilute aqueous SA solutions using ab initio methods. Particularly, they concluded that for dilute solutions, the presence of the acid does not alter significantly the averaged water structure. Otherwise, Choe et al. ${ }^{13}$ carried out a first principles MD study of aqueous SA solutions at two different concentrations: 0.87 and $18.5 \mathrm{~m}$. Our results at $1.13 \mathrm{~m}$ also agree with those findings at $0.83 \mathrm{~m}$. However, we do not observe the notorious shifting of the first and second $g_{\text {ow-ow }}(r)$ peaks to lower distances that these authors saw at $18.5 \mathrm{~m}$, probably because our solutions are not so concentrated.

\section{A.2 Bisulfate ion-water}

The $g_{O B-O W}(r)$ and $g_{O B-H W}(r)$ partial radial distribution functions have been plotted in Figure 3. At $1.13 \mathrm{~m} g_{\text {OB-ow }}(r)$ exhibits two clear peaks located at 0.29 and $0.50 \mathrm{~nm}$ and one minimum at $0.38 \mathrm{~nm}$. Besides, $g_{\mathrm{OB}-H W}(r)$ shows four maxima located at $0.19,0.34$, 0.41 and $0.56 \mathrm{~nm}$, and three minima at $0.25,0.36$ and $0.47 \mathrm{~nm}$. Vchirawongkwin et al. ${ }^{17}$ obtained similar results in their ab initio simulation study of a bisulfate ion in water. Furthermore, Ishiyama and Morita ${ }^{12}$ and Choe et al. ${ }^{13}$ got similar findings in their classical and first principles MD study of aqueous SA solutions. Also, note that the $g_{O B}$ ow $(r)$ and $g_{O B-H W}(r)$ peaks become more significant when the concentration rises. 


\section{A.3 Sulfate dianion-water}

The $g_{\text {os-Ow }}(r)$ and $g_{\text {OS-Hw }}(r)$ have also been displayed in Figure 3. Both radial distribution functions are very similar to the $g_{\text {OB-OW }}(r)$ and $g_{O B-H W}(r)$ ones. Then, at 1.13 $\mathrm{m}$, the $g_{\text {OS-Ow }}(r)$ shows three clear peaks located at 0.28 and 0.49 and $0.67 \mathrm{~nm}$ and two minima at 0.37 and $0.545 \mathrm{~nm}$. On the other hand, $g_{O S-H w}(r)$ exhibits four maxima located at $0.18,0.32,0.4$ and $0.55 \mathrm{~nm}$, and three minima at $0.25,0.36$ and $0.46 \mathrm{~nm}$. These results are consistent with those obtained by Wann et al. ${ }^{14}$ and Ishiyama and Morita $^{12}$ in their MD simulations of dilute aqueous SA solutions using ab initio and classical methods. Also, Vchirawongkwin et al. ${ }^{16}$ and Cannon et al. ${ }^{44}$ reported similar results in their simulation study of a sulfate dianion in water. It should be noticed that gos-ow $(r)$, calculated by Cannon et $a^{4}{ }^{44}$, shows a double peak structure at 0.42 and 0.5 nm, which they attributed to solvation effects, and which we have not found. Finally, note that the peaks of both partial radial distribution functions become less relevant when the acid concentration increases.

\section{A.4 Hydronium-water}

The $g_{\text {O3-OW }}(r)$ and $g_{\text {H3-OW }}(r)$ radial distribution functions have been displayed in Figure 3. At $1.13 \mathrm{~m} g_{\mathrm{H3-O}}(r)$ shows four maxima located at $0.165,0.30,0.40$ and $0.56 \mathrm{~nm}$, and three minima at $0.22,0.345$ and $0.47 \mathrm{~nm}$. Also, $g_{\text {O3-Ow }}(r)$ shows three clear peaks located at 0.26 and 0.475 and $0.69 \mathrm{~nm}$ and two minima at 0.365 and $0.56 \mathrm{~nm}$. It should be noted that the main characteristics of both $g_{\text {O3-Ow }}(r)$ and $g_{\text {ow-Ow }}(r)$ radial distribution functions are very similar to each other. Ishiyama and Morita ${ }^{12}$ and Choe et al. ${ }^{13}$ found similar results in their classical MD and ab initio studies of aqueous SA solutions. At last, it should be noticed that the $g_{\text {O3-OW }}(r)$ and $g_{\text {H3-OW }}(r)$ peak's intensity decreases when the acid concentration rises. 


\section{B. Self-diffusion coefficients}

The mean square displacements of the molecular species at $1.13 \mathrm{~m}$ have been plotted in Figure 4. The self-diffusion coefficients $D_{i}$ have been calculated from the long-time slope of the mean square displacements, using the Einstein relation. ${ }^{34}$ Then, the following relation between the diffusion coefficients have been found: $D_{\mathrm{H}_{2} \mathrm{O}}>$ $D_{\mathrm{HSO}_{4}^{-}}>D_{\mathrm{H}_{3} \mathrm{O}^{+}}>D_{\mathrm{SO}_{4}^{2-}}$. That is to say, the water molecules and the sulfate dianions are, respectively, those which diffuse more and less. The same tendency has been observed for all the concentrations, as can be observed in Table VII, where the selfdiffusion coefficients of all molecular species in terms of the concentration have been collected.

Note that $D_{\mathrm{H}_{2} \mathrm{O}}$ decreases when the SA concentration rises. It should also be noticed that the diffusion coefficient at $1.13 \mathrm{~m}$ is very close to the value reported by Mark and Nilsson $^{42} 2.76 \times 10^{-9} \mathrm{~m}^{2} / \mathrm{s}$ in their MD simulations of pure water using the SPCE model. Wan et al. ${ }^{14}$ also found similar results for dilute SA solutions using a first principles methodology. The self-diffusion coefficient of the hydronium ions $D_{\mathrm{H}_{3} \mathrm{O}^{+}}$at $1.13 \mathrm{~m}$ is $0.80 \times 10^{-9} \mathrm{~m}^{2} / \mathrm{s}$. This result agrees well with that reported by Chen et al. ${ }^{45}(0.76+/-$ $0.22) \times 10^{-9} \mathrm{~m}^{2} / \mathrm{s}$ in their ab initio study of the diffusivity of hydronium and hydroxide ions in liquid water. Unfortunately, to our knowledge, no experimental data of the diffusion coefficients of the bisulfate ions and the sulfate dianions have been found in the literature.

\section{Analysis of hydrogen bonds.}

Two different methodologies, based on energetic and geometric criteria, are generally used to state that two molecules are hydrogen bonded. ${ }^{46,47}$ In this work we have employed the well-known geometric definition. Then, we say that two molecular species form a hydrogen bond (HB) if the intermolecular oxygen-oxygen distance is 
smaller than the first minimum of the oxygen-oxygen radial distribution function, the intermolecular hydrogen-oxygen distance is smaller than the first minimum of the hydrogen-oxygen radial distribution function and the angle between the intermolecular oxygen-oxygen distance and the intermolecular oxygen-hydrogen bond is smaller than $30^{\circ}$.

In this study we have only considered the HB which involve water molecules. Therefore, four different HB types have been analyzed: water-water, bisulfate ionwater, sulfate dianion-water and hydronium-water. In all cases we have employed the cut-off distances corresponding to the first minima of the radial distribution functions depicted in Figure 3. An orientative picture of the four different HB types has been displayed in Figure 5. In the first case, the oxygen atoms of both water molecules can be either HB acceptors or donors. In the second and third types, every water oxygen behaves as an HB donor and the oxygens of both anions as acceptors. In the last case, we consider that every hydronium oxygen acts as a donor and the water oxygen as an acceptor. In all cases the mean number $<n>$ of $\mathrm{HB}$ and the percentages $f_{n}$ of molecules or ions bonded to " $n$ ” water molecules have been computed. The results are displayed, respectively, in Table VIII and Figure 6.

The water-water HB results at $1.13 \mathrm{~m}$ are $<n>=3.2, f_{2}=17, f_{3}=35.5$ and $f_{4}=39$. These findings are very similar to those obtained by Padró et al. ${ }^{48}$ in their MD study of pure water $<n>=3.3, f_{2}=13.5, f_{3}=37.5$ and $f_{4}=42$. Moreover, the characteristic branched network of $\mathrm{HB}$ is notably modified when the concentration rises. More precisely, $<n>$ and the percentages $f_{3}$ and $f_{4}$ diminish and, at the same time, $f_{2}$ increases. This phenomenon becomes more and more noticeable as the SA concentration increases. Then, the results at $6.79 \mathrm{~m}$ are $<n>=2.3, f_{2}=30, f_{3}=28$ and $f_{4}=15$. That is, as the ionic population increases, the water molecules tend to bond to the ions. 
The average numbers $<n>$ of bisulfate-water HB are gathered in Table VIII. These results show that, on average and regardless of the acid concentration, more than six water molecules are hydrogen bonded to every bisulfate ion and that $<n>$ slightly decreases when the acid concentration rises. Furthermore, Figure 6 shows that at $1.13 \mathrm{~m}$ the percentages of bisulfate ions bonded with " $n$ ” water molecules are: $f_{5}=14.5, f_{6}=$ 27.5, $f_{7}=25.5$ and $f_{8}=17$. An increase of the acid concentration gives rise to a decrease in the number of bisulfate ions hydrogen bonded to more than five water molecules, Then, at $6.79 \mathrm{~m}$ the percentages are $f_{5}=21.5, f_{6}=19.5, f_{7}=13$ and $f_{8}=6$.

At dilute solutions the sulfate dianions are hydrogen bonded to a large number of water molecules. According to Table VIII and Figure 6, we have the following results at $1.13 \mathrm{~m}:<n>=7$ and $f_{5}=13, f_{6}=14.5, f_{7}=19, f_{8}=19$ and $f_{9}=13$. Besides, an increase of the acid concentration gives rise to a strong reduction of the number of water molecules hydrogen bonded to every sulfate dianion. Then, at $6.79 \mathrm{~m}$ we obtain the following results $<n>=3$ and $f_{5}=8, f_{6}=5, f_{7}=2, f_{8}=1$ and $f_{9}=0.5$. To sum up, the number of water molecules hydrogen bonded to every sulfate dianion decreases when the concentration rises, being these changes more noticeable than those observed for the bisulfate ions.

According to the ab initio study of Chen et $a .^{45}$, on the diffusivity of hydroxide and hydronium ions in water, in absence of proton transfer the number of HB donors of a hydronium ion is 1.0 per proton, and the number of $\mathrm{HB}$ acceptors is 0.35 per lone electron pair. These results differ from those found when the water-water HB structure is studied. In this last case the authors stated that the number of HB acceptors per lone electron pair and of HB donors per proton were in both cases 0.87 . Therefore, it can be assumed that on average every hydronium ion donates three $\mathrm{HB}$ and it practically accepts none. In addition, the average number of HB around a water molecule is larger 
than that found for every hydronium ion. The data summarized in Table VIII corroborate these findings. Then, the results at $1.13 \mathrm{~m}$ are $<n>=2.1$ and $f_{2}=48.5$ and $f_{3}$ $=34$. Finally, a notorious reduction of the number of water molecules hydrogen bonded to every hydronium ion has been observed when the SA concentration rises. Then, the results at $6.79 \mathrm{~m}$ are $<n>=1.4$ and $f_{2}=28$ and $f_{3}=15$.

Now we turn to the study of the dynamics of formation and rupture of HB. Following the ideas exposed by Luzar ${ }^{49}$, the HB lifetimes can be computed using two methodologies, according to what she defines as the non-interrupted and interrupted lifetimes. In the non-interrupted, continuous or fast case, every HB always remains intact. Its lifetime $\tau_{C H B}$ is the time integral of the probability $P(t)$ that a pair of molecules, initially bonded, remains bonded at all times up to time $t . P(t)$ is obtained making a histogram of the HB lifetimes for each configuration.

In the interrupted or slow lifetime case, every HB can break and re-form. The lifetime is computed by defining the probability $c(t)$ that two molecules are bonded at time $t$, provided that this pair was bonded at $t=0$. Then, $c(t)$ is defined from the following equation:

$$
c(t)=<h(t) h(0)>/<h(0)^{2}>.
$$

$h(t)$ is a binary function: $h(t)=1$ if molecules “i,j" are bonded at time $t$ and $h(t)=0$ if are not bonded. $c(t)$ shows an exponential behavior, followed by a power law tail. The $\tau_{c}$ lifetime is computed from the $c(t)$ time integral.

It is well-known that the self-diffusion of the molecules leads to smaller $\mathrm{HB}$ relaxation time values ${ }^{50}$. This problem can be circumvented introducing the following correlation function: ${ }^{49}$

$$
n(t)=\langle h(0)[1-h(t)] H(t)>/<h(0)>,
$$


where $H(t)=1$ if the oxygen-oxygen distance between the pair of molecules “i,j” is closer than the first minimum of $g_{O-O}(r)$ at time $t$ and $H(t)=0$ otherwise. Then, the dynamics of the HB is defined using the reactive flux correlation function $k(t)=$ $d c(t) / d t$, which according to the Luzar and Chandler theory, ${ }^{49}$ is related to $n(t)$ and $c(t)$ by the following equation:

$$
k(t)=k c(t)-k^{\prime} n(t),
$$

where $k$ and $k$ ' are the rate constants associated to the processes of break and reformation of the HB. It is well-known that at intermediate times $k(t)$ decays following an exponential behavior, which is generally used to compute the interrupted or slow lifetime $\tau_{\text {IHB }}$

The continuous $\tau_{C H B}$ and the interrupted $\tau_{I H B}$ lifetime values are gathered in Table IX. At $1.13 \mathrm{~m}$ the water-water HB lifetimes are very similar to those found by Starr et al. ${ }^{51}\left(\tau_{C H B}=0.27 \mathrm{ps}\right.$ and $\left.\tau_{\text {IHB }}=5.18 \mathrm{ps}\right)$ in their classical MD study of pure water using the SPCE model and those of Guàrdia et al. ${ }^{52}$ using the Car-Parrinello method $\left(\tau_{C H B}=0.46 \mathrm{ps}\right.$ and $\left.\tau_{\mathrm{IHB}}=4.90 \mathrm{ps}\right)$.

The water-water and the hydronium-water $\tau_{C H B}$ values are larger than the two anion-water continuous lifetimes, suggesting that the water molecules hydrogen bonded to the anions are more labile than those bonded to the hydronium ions or to other water molecules. The three ion-water continuous lifetimes do not significantly change when the acid concentration rises. However, the water-water ones clearly decrease. Therefore, we can conclude that a more labile behavior of the water molecules, which are hydrogen bonded to other water molecules, has been observed. The hydronium-water $\tau_{C H B}$ and $\tau_{I H B}$ lifetimes are larger than the water-water HB ones, denoting that the hydroniumwater HB are stronger than the water-water ones. This phenomenon can also be explained in terms of the hydrogen-oxygen radial distribution functions. Indeed, note 
the differences between the first peak heights of the $g_{H W-O W}(r)$ and $g_{H 3-O W}(r)$, depicted in Figure 3.

The sulfate dianion-water $\tau_{I H B}$ interrupted lifetime values are larger than the bisulfate ion-water ones. Moreover, it should be noted that in all cases as bigger is the acid concentration larger are their interrupted HB lifetimes. Again, we observe how an increase of the acid concentration leads to a partial breaking of the tetrahedral structure of water, basically because, as we have previously mentioned, the water molecules will prefer to bond to the ions. As a result, there is a reduction on the percentages of water molecules hydrogen bonded to other four water molecules $f_{4}$ and an increase of $f_{1}$ and $f_{2}$, and their interrupted lifetime $\tau_{I H B}$ values. At the same time, there is a reduction on the percentages $f_{n}$ of ions hydrogen bonded with a large " $n$ " number of water molecules (n $>6$ for $\mathrm{HSO}_{4}^{-}-\mathrm{H}_{2} \mathrm{O}$ and $\mathrm{SO}_{4}^{2-}-\mathrm{H}_{2} \mathrm{O}$, and $\mathrm{n}>2$ for $\mathrm{H}_{3} \mathrm{O}^{+}-\mathrm{H}_{2} \mathrm{O}$ ) and an increase of the interrupted lifetimes in all the three ion-water HB types, which is especially notorious in the sulfate dianion-water case.

\section{CONCLUSIONS}

MD simulations of aqueous SA solutions at room temperature have been performed. The SA dissociation has been implicitly taken into account by assuming that the simulated systems are made up of the following four molecular constituent species: water molecules, hydronium cations, bisulfate anions and sulfate dianions. In a first step, MD simulations have been devoted to look for a force field which provides a good agreement between the experimental and the simulated density and viscosity coefficients in a range of concentrations up to $40 \%$ wt. Afterwards, MD simulations have been used to compute the radial distribution functions, which involve water molecules, and the diffusion coefficient of the constituent species. We have found that 
the water molecules and the bisulfate dianions are, respectively, those which diffuse more and less.

A hydrogen bond analysis, which involves water molecules, based on the geometric criteria has also been performed. Then, the mean number $<n>$ of $\mathrm{HB}$, the percentages $f_{n}$ of molecular species (water, bisulfate ion, sulfate dianion, or hydronium ion) bonded with " $n$ " water molecules, the continuous $\tau_{C H B}$ and interrupted $\tau_{I H B}$ lifetimes have also been calculated. At low dilutions the structure of the water molecules, which are hydrogen bonded to other water molecules, is very similar to that of pure water. Likewise, the water-water $<n>$ value is larger than the hydronium-water one and much lower than those of both bisulfate anion-water and sulfate dianion-water. At the same time, the water molecules hydrogen bonded to the anions are more labile. On the other hand, an increase of the acid concentration gives rise to a growth of the interrupted lifetimes and to a partial breaking of the water HB network around every water molecule, so $<n>$, $f_{3}$, and $f_{4}$ decrease. Finally, the number of water molecules, hydrogen bonded to every sulfate dianion, strongly decreases and those around every hydronium ion and bisulfate anion diminishes more slowly.

\section{ACKNOWLEDGEMENTS}

Financial support from the Ministerio de Economía y Competitividad of Spain, Grant FIS2015-66879-C2-1-P (MINECO/FEDER) is gratefully acknowledged. 


\section{REFERENCES}

${ }^{1}$ P. J. Chenier, Survey of Industrial Chemistry (Springer, New York, 2002).

${ }^{2}$ M. F. Fingas, Handbook of Hazardous Materials Spills Technology (McGraw-Hill, New York, 2002).

${ }^{3}$ K. I. Popov, S. S. Djokić, B. N. Grgur, Electrowinning. in: Fundamental Aspects of Electrometallurgy (Springer, Boston, MA, 2002).

${ }^{4}$ S. C. Levy and P. Bro, Battery of Hazards and Accidents Prevention (Plenum Press, New York, 1994).

${ }^{5}$ K. S. Carslaw, T. Peter and S. L. Clegg, Rev. Geophys. 35,125 (1997).

${ }^{6}$ D. A. Knopf, B. P. Luo, U. K. Krieger and T. Koop, J. Phys. Chem. A 107, 4322 (2003).

${ }^{7}$ I. Kusaka, Z. G. Wang and J. H. Seinfeld, J. Chem. Phys. 108, 6829 (1998).

${ }^{8}$ S. M. Kathmann and B. N. Hale, J. Phys. Chem. B 105, 11719 (2001).

${ }^{9}$ C.-G. Ding, T. Taskila, K. Laasonen and A. Laaksonen, Chem. Phys. 287,7 (2003).

${ }^{10}$ M. Toivola, I. Napari and H. Vehkamäki, Boreal Environment Research 14, 654 (2009).

${ }^{11}$ H. Matsubara, T. Ebisuzaki and K. Yasuoka, J. Chem. Phys. 130, 104705 (2009).

${ }^{12}$ T. Ishiyama and A. Morita, J. Phys. Chem. C 115, 13704 (2011).

${ }^{13}$ Y.-K. Choe, E. Tsuchida and T. Ikeshoji, J. Chem. Phys. 126, 154510 (2007).

${ }^{14}$ Q. Wann, L. Spanu, F. Gygi and G. Galli, J. Phys. Chem. Lett. 5, 2562 (2014).

${ }^{15}$ A. D. Hammerich, V. Buch and F. Mohamed, Chem. Phys. Lett. 460, 423 (2008).

${ }^{16}$ V. Vchirawongkwin, B. M. Rode and I. Persson, J. Phys. Chem. B 111, 4150 (2007).

${ }^{17}$ V. Vchirawongkwin, C. Kritayakornupong and B. M. Rode, J. Phys. Chem. B 114, $11561(2010)$. 
${ }^{18}$ G. A. Jeffrey, An Introduction to Hydrogen Bonding (Oxford University Press, New York, 1997).

${ }^{19}$ S. J. Grabowski (ed), Hydrogen Bonding-New Insights (Springer, Dordrecht, 2006).

${ }^{20}$ B. M. Ladanyi and M. S. Skaf, Annu. Rev. Phys. Chem. 44, 335 (1993).

${ }^{21}$ Y. Marechal, The Hydrogen Bond and the Water Molecule: The Physics and Chemistry of Water, Aqueous and Bio-Media (Elsevier, Amsterdam, 2007).

${ }^{22}$ M. Canales and E. Guàrdia, J. Mol. Liq. 224, 1064 (2016).

${ }^{23}$ H. Arstila, K. Laasonen and A. Laaksonen, J. Chem. Phys. 108, 1031 (1998).

${ }^{24}$ H. J. C. Berendsen, J. R. Grigera and T. P. Straatsma, J. Phys. Chem. 91, 6269 (1987).

${ }^{25}$ B. Hess, C. Kutzner and D. van der Spoel, J. Chem. Theory Comput. 4, 435 (2008).

${ }^{26}$ J. P. Ryckaert, G. Cicotti and H. J. C. Berendsen, J. Comput. Phys. 23, 327 (1977).

${ }^{27}$ W. L. Jorgensen, D. S. Maxwell and J. Tirado-Rives, J. Am. Chem. Soc. 118, 11225 (1996).

${ }^{28}$ M. H. Ketko, G. Kamath and J. J. Potoff, J. Phys. Chem. B 115, 4949 (2011).

${ }^{29}$ J. Yosa and M. Meuwly, J. Phys. Chem. A 115, 14350 (2011).

${ }^{30}$ O. Teleman, B. Jönsson and S. Engström, Mol. Phys. 60, 193 (1987)

${ }^{31}$ J. P. Ryckaert and A. Bellemans, Chem. Phys. Lett. 30, 123 (1975).

${ }^{32}$ B. Hess, C. Kutzner and D. van der Spoel, J. Chem. Theory Comput. 4, 435 (2008).

${ }^{33}$ H. J. C. Berendsen, J. P. M. Postma, W. F. van Gunsteren, A. DiNola and J. R. Haak, J. Chem. Phys. 81, 3684 (1984).

${ }^{34}$ M. P. Allen and D. J. Tildesley, Computer Simulation of Liquids (Oxford University Press, New York, 1987).

${ }^{35}$ T. Darden, D. York and L. Pedersen, J. Chem. Phys. 98, 10089 (1993).

${ }^{36}$ A. R. Leach, Molecular Modelling: Principles and Applications (Pearson, Harlow, 2001). 
${ }^{37}$ R. H. Perry, D. W. Green and J. O. Maloney, Perry’s Chemical Engineers' Handbook $7^{\text {th }}$ ed. (McGraw-Hill, New York, 1997).

${ }^{38}$ G. W. Vinal and D. N. Craig, Bureau of Standards Journal Research, 10, 781 (1933). Research Paper 566.

${ }^{39}$ J. Kestin, M. Sokolov and W. A. Wakeham, J. Phys. Chem. Ref. Data, 7, 941 (1978).

${ }^{40}$ B. Hess, J. Chem. Phys. 116, 209 (2002).

${ }^{41}$ M. A. González and J. L. F. Abascal, J. Chem. Phys., 132, 096101 (2010).

${ }^{42}$ P. Mark and L. Nilsson, J. Phys. Chem. A, 105, 9954 (2001).

${ }^{43}$ P. Gallo, D. Corradini and M. Rovere, J. Mol. Liq. 189, 52 (2014).

${ }^{44}$ W. R. Cannon, B. M. Pettitt and J. A. McCammon, J. Phys. Chem., 98, 6225 (1994).

${ }^{45}$ M. Chen, L. Zheng, B. Santra, H.-Y. Ko, R. A. DiStasio Jr, M. L. Klein, R. Car and X. Wu, Nat. Chem., 10, 413 (2018).

${ }^{46}$ D. Bertolini, M. Cassettari, M. Ferrario, P Grigolini and G. Salvetti, Adv. Chem. Phys.62, 277 (1985).

${ }^{47}$ F. Sciortino and S. L. Fornili, J. Chem. Phys. 90, 2786 (1989).

${ }^{48}$ J. A. Padró, L. Saiz and E. Guàrdia J. Mol. Struct 416, 243 (1997).

${ }^{49}$ A. Luzar J. Chem. Phys. 113, 10663 (2000).

${ }^{50}$ H. S. Lee and M. E. Tuckerman, J. Chem. Phys. 126, 164501 (2007).

${ }^{51}$ F. W. Starr, J. K. Nielsen and H. E. Stanley, Phys. Rev. E 62, 579 (2000).

${ }^{52}$ E. Guàrdia, I. Skarmoutsos, M. Masia, J. Phys. Chem. B 119, 8926 (2015). 


\section{TABLES}

TABLE I: Bond lengths (nm).

\begin{tabular}{|c|c|c|c|c|}
\hline Bond & $\mathrm{HSO}_{4}^{-}$ & $\mathrm{SO}_{4}^{2-}$ & $\mathrm{H}_{3} \mathrm{O}^{+}$ & $\overline{\mathrm{H}} \mathrm{H}_{2} \mathrm{O}$ \\
\hline "S-O & $0.1690^{\mathrm{a}}$ & - & - & - \\
\hline S-O' & $0.1490^{\mathrm{a}}$ & $0.1527^{\mathrm{b}}$ & - & - \\
\hline $\mathrm{H}-\mathrm{O}$ & $0.1000^{\mathrm{a}}$ & - & $0.0973^{\mathrm{C}}$ & $0.1000^{d}$ \\
\hline
\end{tabular}

TABLE II: Equilibrium bond angles $\theta_{e q}$ (degrees) and harmonic force constants $k_{\theta}$ $\left(\mathrm{kJmol}^{-1} \mathrm{rad}^{-2}\right)$.

\begin{tabular}{cccccccc}
\hline & \multicolumn{2}{c}{$\mathrm{HSO}_{4}^{-}$} & \multicolumn{2}{c}{$\mathrm{SO}_{4}^{2-}$} & \multicolumn{2}{c}{$\mathrm{H}_{3} \mathrm{O}^{+}$} & $\mathrm{H}_{2} \mathrm{O}$ \\
Bond & $\theta_{e q}$ & $k_{\theta}$ & $\theta_{e q}$ & $k_{\theta}$ & $\theta_{e q}$ & $k_{\theta}$ & $\theta_{e q}$ \\
\hline $\mathrm{H}-\mathrm{O}-\mathrm{S}$ & $106.50^{\mathrm{a}}$ & $370^{\mathrm{a}}$ & - & - & - & - & - \\
$\mathrm{O}-\mathrm{S}-\mathrm{O}$, & $104.20^{\mathrm{a}}$ & $400^{\mathrm{a}}$ & - & - & - & - & - \\
$\mathrm{O}-\mathrm{S}-\mathrm{O}$, & $114.10^{\mathrm{a}}$ & $460^{\mathrm{a}}$ & $109.5^{\mathrm{a}}$ & $460^{\mathrm{a}}$ & - & - & - \\
$\mathrm{H}-\mathrm{O}-\mathrm{H}$ & - & - & - & - & $111.6^{\mathrm{b}}$ & $383^{\mathrm{b}}$ & $109.47^{\mathrm{c}}$ \\
\hline \hline
\end{tabular}

\footnotetext{
${ }^{\mathrm{a}}$ Reference 22 .

${ }^{\mathrm{b}}$ Reference 30.

${ }^{\mathrm{c}}$ Reference 24.
} 
TABLE III: Ryckaert-Bellemans (Reference 31) H-O-S-O' dihedral torsional potential parameters in $\mathrm{kJmol}^{-1}$.

\begin{tabular}{ccccccc}
\hline \hline Dihedral & $C 0$ & $C 1$ & $C 2$ & $C 3$ & $C 4$ & C5 \\
\hline \hline H-O-S-O' & 1.8 & -5.4 & 0 & 7.2 & 0 & 0 \\
& & & & & & \\
\hline \hline
\end{tabular}

TABLE IV: Lennard-Jones parameters ( $\sigma$ in nm and $\varepsilon$ in $\mathrm{kJmol}^{-1}$ )

\begin{tabular}{|c|c|c|c|c|c|c|}
\hline \multirow[b]{2}{*}{ Atom } & \multicolumn{2}{|c|}{$\mathrm{HSO}_{4}^{-}$} & \multicolumn{2}{|c|}{$\mathrm{SO}_{4}^{2-}$} & \multicolumn{2}{|c|}{$\mathrm{H}_{3} \mathrm{O}^{+}$and $\mathrm{H}_{2} \mathrm{O}$} \\
\hline & $\sigma$ & $\varepsilon$ & $\sigma$ & $\varepsilon$ & $\sigma$ & $\varepsilon$ \\
\hline $\mathrm{S}$ & $0.355^{\mathrm{a}}$ & $1.0465^{\mathrm{a}}$ & $0.355^{\mathrm{a}}$ & $1.0465^{\mathrm{a}}$ & - & - \\
\hline $\mathrm{O}^{\prime}$ & $0.315^{\mathrm{a}}$ & $0.8371^{\mathrm{a}}$ & $0.315^{\mathrm{a}}$ & $0.8371^{\mathrm{a}}$ & - & - \\
\hline $\mathrm{O}$ & $0.315^{\mathrm{a}}$ & $0.8371^{\mathrm{a}}$ & - & - & $0.316557^{b}$ & $0.650194^{\mathrm{b}}$ \\
\hline $\mathrm{H}$ & $0^{\mathrm{a}}$ & $0^{\mathrm{a}}$ & - & - & $0^{\mathrm{b}}$ & $0^{\mathrm{b}}$ \\
\hline
\end{tabular}

TABLE V: Simulation details: Molality m (mol/kg), wt \%, dissociation constant $\alpha$ (Reference 6), number of molecules of every constituent species.

\begin{tabular}{ccccccc}
\hline \hline$m$ & $w t \%$ & $\alpha$ & $\mathrm{HSO}_{4}^{-}$ & $\mathrm{SO}_{4}^{2-}$ & $\mathrm{H}_{3} \mathrm{O}^{+}$ & $\mathrm{H}_{2} \mathrm{O}$ \\
\hline \hline 1.13 & 10 & 0.37 & 13 & 7 & 27 & 973 \\
2.55 & 20 & 0.42 & 27 & 19 & 65 & 935 \\
4.37 & 30 & 0.40 & 47 & 32 & 111 & 889 \\
6.79 & 40 & 0.41 & 72 & 50 & 172 & 828 \\
\hline \hline
\end{tabular}


TABLE VI: Partial charges (atomic units)

\begin{tabular}{ccccc}
\hline \hline Atom & $\mathrm{HSO}_{4}^{-}$ & $\mathrm{SO}_{4}^{2-}$ & $\mathrm{H}_{3} \mathrm{O}^{+}$ & $\mathrm{H}_{2} \mathrm{O}$ \\
\hline \hline $\mathrm{S}$ & 0.6 & 0.6 & - & - \\
$\mathrm{O}$ & -0.45 & -0.65 & - & - \\
$\mathrm{O}$ & -0.50 & - & -0.248 & $-0.8476^{\mathrm{a}}$ \\
$\mathrm{H}$ & 0.25 & - & 0.416 & $0.4238^{\mathrm{a}}$ \\
\hline \hline
\end{tabular}

${ }^{\mathrm{a}}$ Reference 24.

TABLE VII: Experimental and simulation results of the density $\rho\left(\mathrm{gcm}^{-3}\right)$ (Reference 37) and viscosity $\eta$ (cP) (Reference 38). Self-diffusion coefficients $D\left(10^{-9}\right.$ $\mathrm{m}^{2} / \mathrm{s}$ ) of water and ions.

\begin{tabular}{ccccccccc}
\hline \hline$w t \%$ & $\rho_{\text {sim }}$ & $\rho_{\text {exp }}$ & $\eta_{\text {sim }}$ & $\eta_{\text {exp }}$ & $D_{\mathrm{H}_{2} \mathrm{O}}$ & $D_{\mathrm{HSO}_{4}^{-}}$ & $D_{\mathrm{SO}_{4}^{2-}}$ & $D_{\mathrm{H}_{3} \mathrm{O}^{+}}$ \\
\hline \hline 10 & 1.067 & 1.064 & 0.84 & 1.09 & 2.35 & 1.33 & 0.29 & 0.77 \\
20 & 1.144 & 1.137 & 1.10 & 1.37 & 1.99 & 1.10 & 0.14 & 0.53 \\
30 & 1.228 & 1.215 & 1.83 & 1.78 & 1.63 & 0.75 & 0.12 & 0.46 \\
40 & 1.321 & 1.299 & 3.57 & 2.41 & 1.21 & 0.43 & 0.08 & 0.27 \\
\hline \hline
\end{tabular}

TABLE VIII: Mean number of HB for different acid concentrations.

\begin{tabular}{ccccc}
\hline \hline$m$ & $\mathrm{H}_{2} \mathrm{O}-\mathrm{H}_{2} \mathrm{O}$ & $\mathrm{HSO}_{4}^{-}-\mathrm{H}_{2} \mathrm{O}$ & $\mathrm{SO}_{4}^{2-}-\mathrm{H}_{2} \mathrm{O}$ & $\mathrm{H}_{3} \mathrm{O}^{+}-\mathrm{H}_{2} \mathrm{O}$ \\
\hline \hline 1.13 & 3.2 & 7.2 & 7.0 & 2.1 \\
2.55 & 3 & 7 & 5.0 & 1.7 \\
4.37 & 2.5 & 6.3 & 4.0 & 1.5 \\
6.79 & 2.3 & 6.1 & 3.0 & 1.4 \\
\hline \hline
\end{tabular}


TABLE IX: Continuous and interrupted HB lifetimes (ps) for different acid concentrations.

\begin{tabular}{cccccccccc}
\hline \hline & $\mathrm{H}_{2} \mathrm{O}-\mathrm{H}_{2} \mathrm{O}$ & $\mathrm{HSO}_{4}^{-}-\mathrm{H}_{2} \mathrm{O}$ & \multicolumn{2}{c}{$\mathrm{SO}_{4}^{2-}-\mathrm{H}_{2} \mathrm{O}$} & \multicolumn{2}{l}{$\mathrm{H}_{3} \mathrm{O}^{+}-\mathrm{H}_{2} \mathrm{O}$} \\
\hline$m$ & $\tau_{C H B}$ & $\tau_{I H B}$ & $\tau_{C H B}$ & $\tau_{\text {IHB }}$ & $\tau_{C H B}$ & $\tau_{I H B}$ & $\tau_{C H B}$ & $\tau_{I H B}$ \\
\hline 1.13 & 0.29 & 6.4 & 0.09 & 4.4 & 0.13 & 9.5 & 0.34 & 14.0 \\
2.55 & 0.27 & 6.5 & 0.08 & 5.0 & 0.11 & 14.8 & 0.33 & 16.9 \\
4.37 & 0.22 & 7.4 & 0.08 & 6.0 & 0.11 & 18.3 & 0.34 & 20.7 \\
6.79 & 0.17 & 9.1 & 0.07 & 7.6 & 0.10 & 24.5 & 0.34 & 24.6 \\
\hline
\end{tabular}




\section{CAPTIONS TO FIGURES}

FIG. 1. Most favorable structures of the water molecules, the hydronium ions $\mathrm{H}_{3} \mathrm{O}^{+}$, the bisulfate ions $\mathrm{HSO}_{4}^{-}$and the sulfate dianions $\mathrm{SO}_{4}^{2-}$. Labels correspond to the atom types defined in Tables I, II, III, IV and VI.

FIG. 2. Shear viscosities at $298 \mathrm{~K}$ for different acceleration amplitudes and acid concentrations.

FIG. 3. Oxygen-oxygen and hydrogen-oxygen radial distribution functions at $298 \mathrm{~K}$ for different acid concentrations. Continuous line: $1.13 \mathrm{~m}$, dotted line: $2.55 \mathrm{~m}$, dashed line $4.37 \mathrm{~m}$ and dotted-dashed line: $6.79 \mathrm{~m}$.

FIG. 4. Mean square displacements of the molecular species at $298 \mathrm{~K}$ and $1.13 \mathrm{~m}$.

FIG. 5. Representative snapshots of the hydronium-water, water-water, bisulfate ionwater and sulfate-dianion-water hydrogen bonds at $1.13 \mathrm{~m}$.

FIG. 6. Histograms with the percentages $f_{n}$ of molecular species (water, bisulfate ion, sulfate dianion, or hydronium ion) bonded with “ $n$ ” water molecules for different acid concentrations. White: $1.13 \mathrm{~m}$, white and dotted: $2.55 \mathrm{~m}$, grey and dashed: $4.37 \mathrm{~m}$ and black: $6.79 \mathrm{~m}$. 


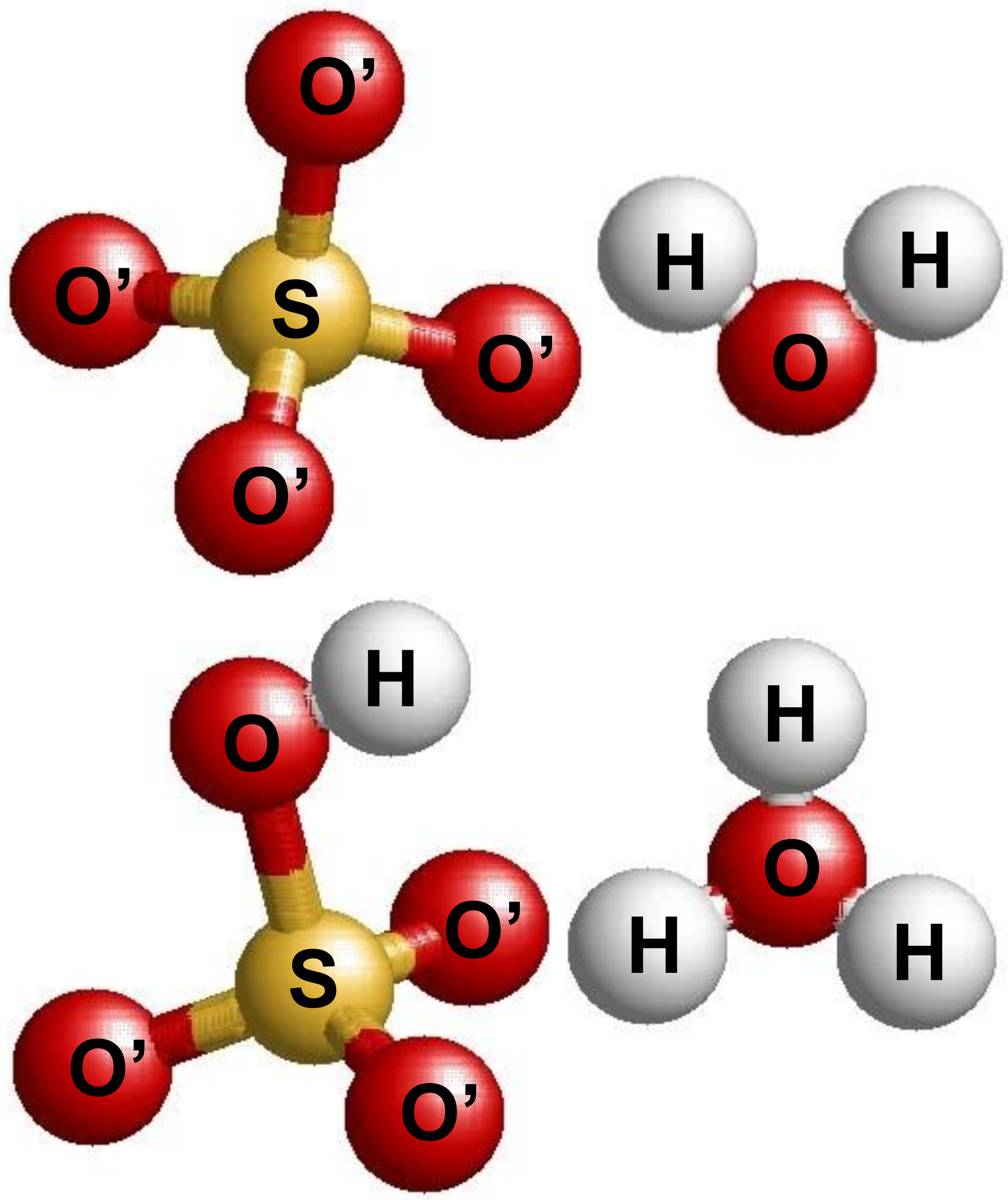

Figure 1 


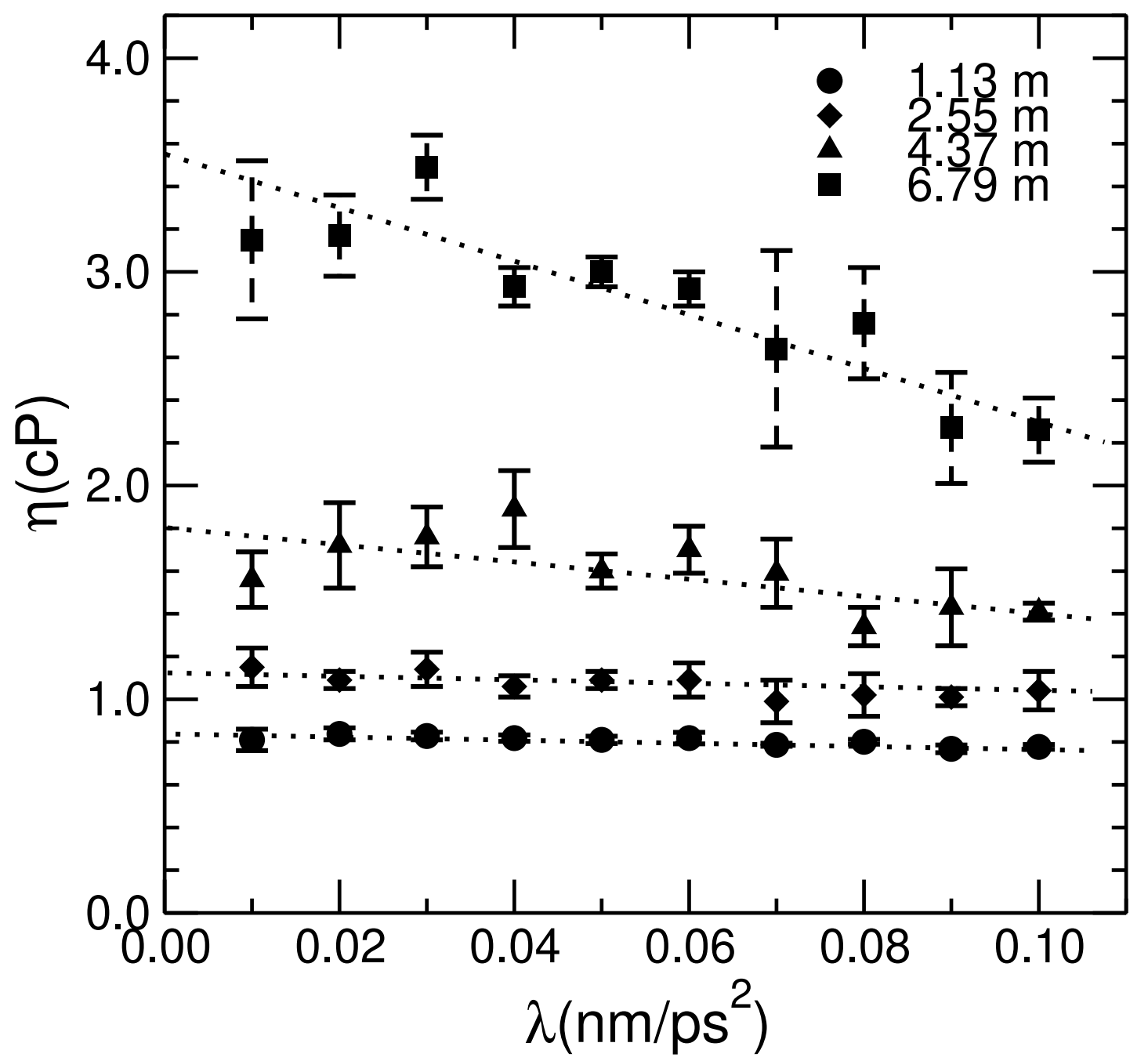

Figure 2 


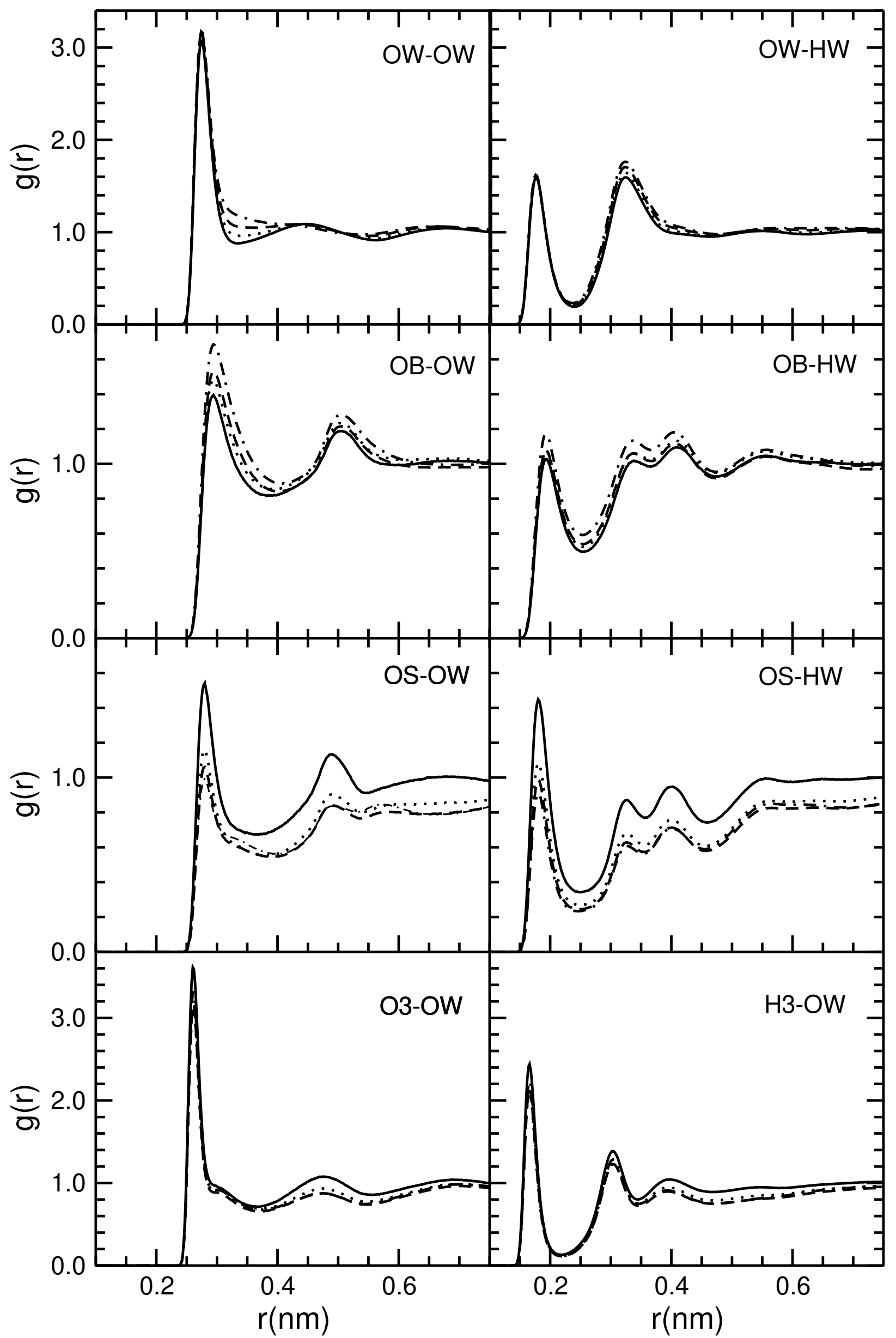

Figure 3 


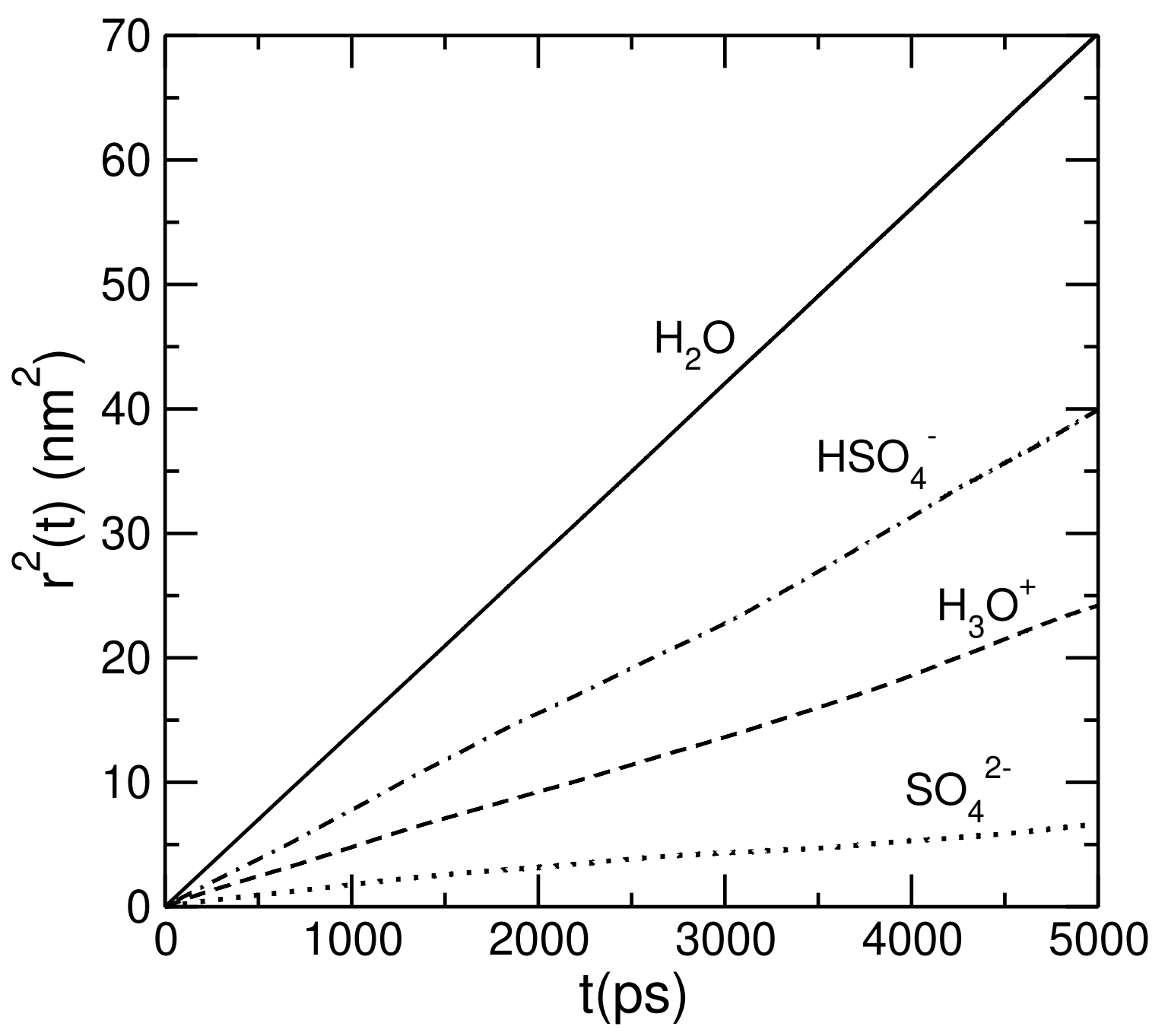

Figure 4 


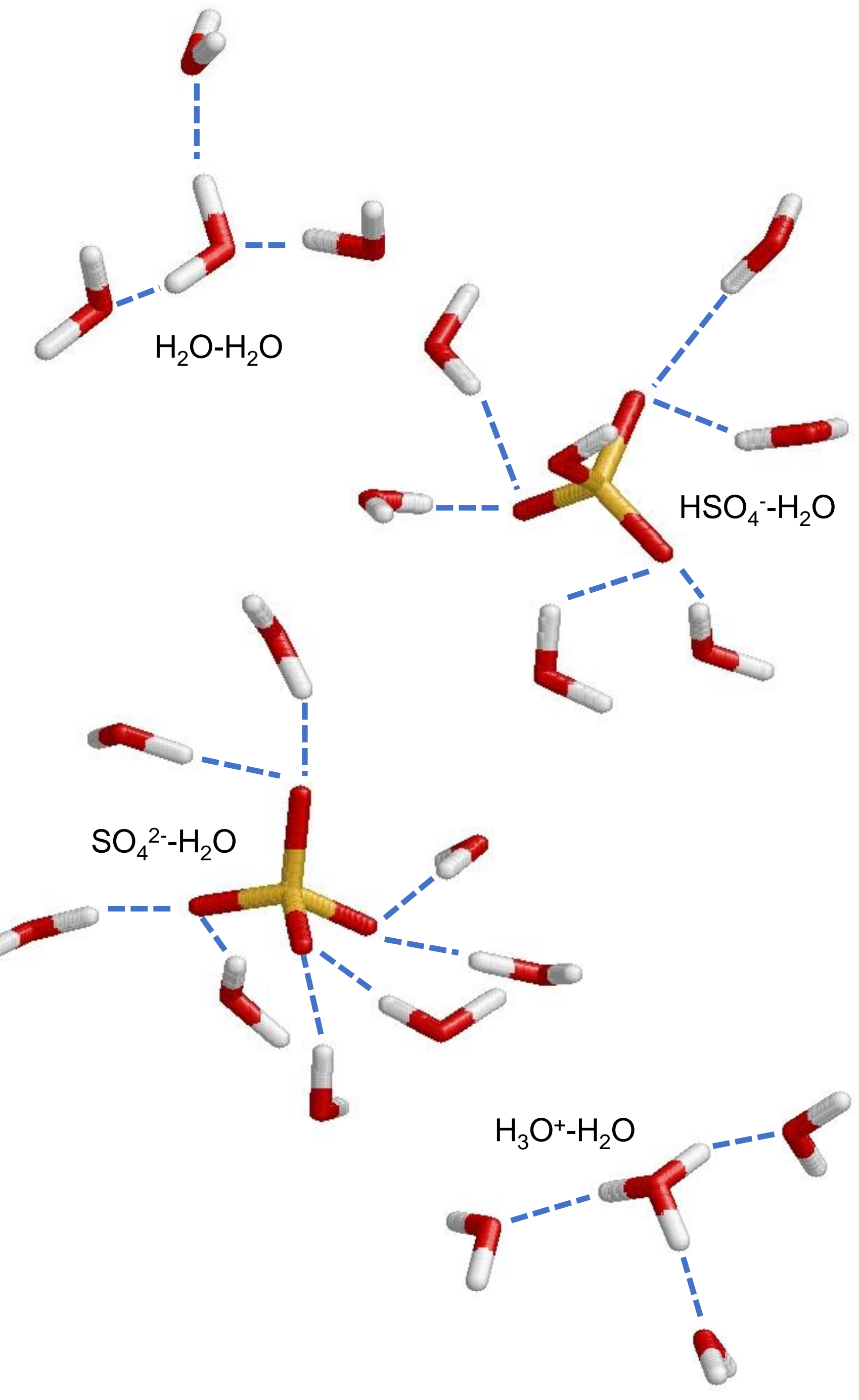

Figure 5 

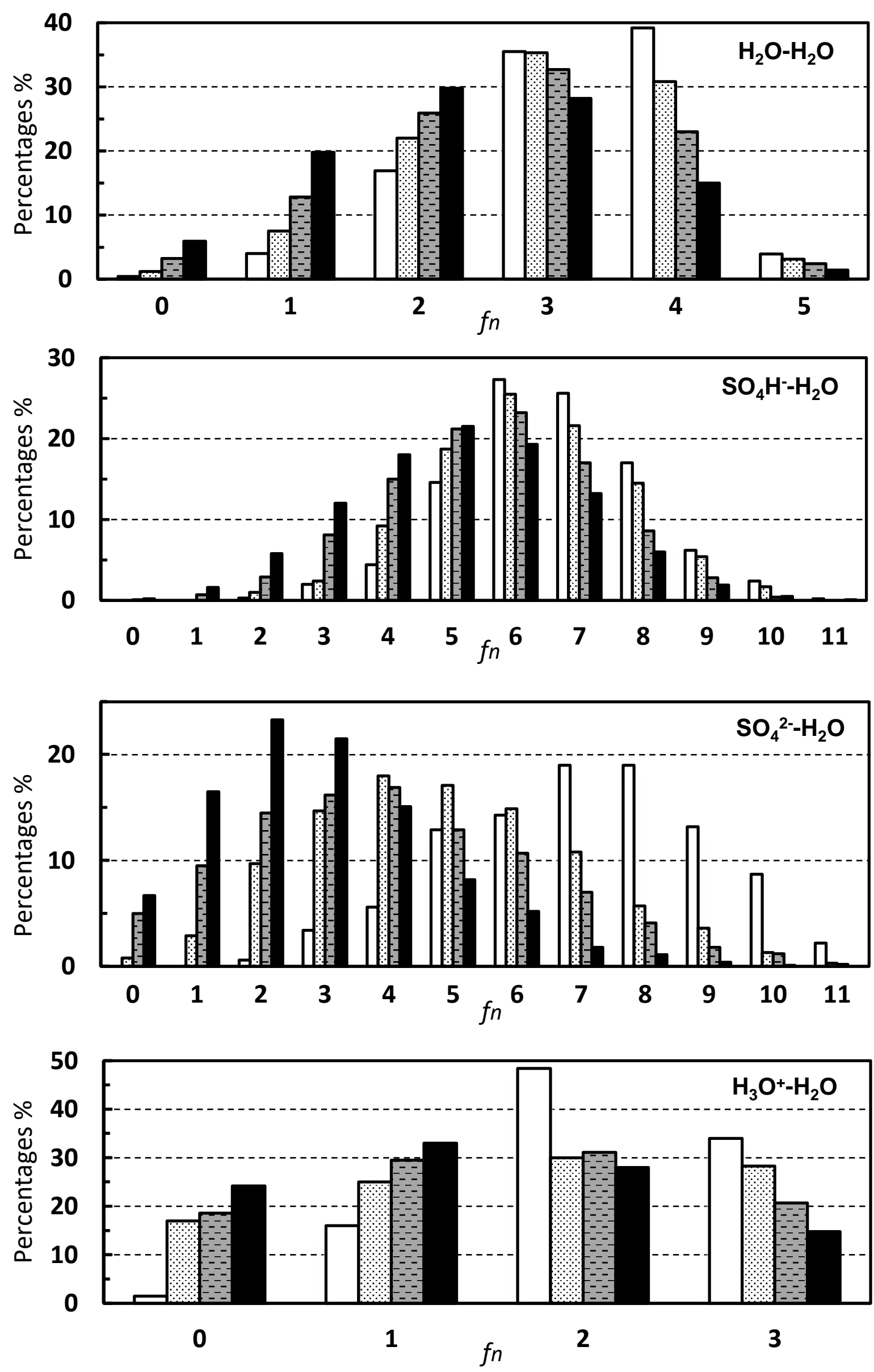

Figure 6 